\title{
Coupling Aging Immunity with a Sedentary Lifestyle: Has the Damage Already Been Done? - A Mini-Review
}

\author{
Richard J. Simpson Keith Guy \\ Laboratory of Integrated Physiology, Department of Health and Human Performance, University of Houston, \\ Houston, Tex., USA
}

\section{Key Words \\ Exercise $\cdot$ Immunosenescence $\cdot$ Telomeres $\cdot$ Immune risk profile $\cdot$ Cytomegalovirus $\cdot$ Physical activity}

\begin{abstract}
The elderly population is at an unprecedented risk of infectious diseases and malignancy due to apparently inevitable age-related declines in immunity. The 'immune risk profile' (IRP) is an array of biomarkers that has been used to predict morbidity and mortality in older adults. As it is generally accepted that middle-aged and elderly individuals who habitually participate in moderate-intensity exercise are less likely to incur an infection than their sedentary counterparts, this review addresses current knowledge on the effects of regular exercise on aspects of adaptive immunity as they relate to the IRP. Findings from cross-sectional studies mostly show enhanced immunity in physically active compared to sedentary older adults. These include greater T-cell responsiveness to mitogens in vitro, a reduced frequency of antigen-experienced and senescent T-cells (i.e. CD45RO+/ KLRG1+/CD57+/CD28-), enhanced IL-2 production and Tlymphocyte expression of the IL-2 receptor, longer chromosome telomere lengths in blood leukocytes and in vivo immune responses to vaccines and recall antigens. In contrast, the evidence from the available longitudinal studies that have used an exercise training intervention in previously
\end{abstract}

sedentary elderly to improve similar immune responses is less compelling. Although this might indicate that exercise has limited immune restorative properties in previously sedentary elderly, there are still relatively few studies that have addressed specific IRP criteria and the large variation in experimental design among the longitudinal studies complicates the juxtaposition of these results. It is clear that a more substantial and focused research approach is required before physical exercise can be used in earnest as an effective immune restorative strategy in the elderly. This mini-review summarizes the major findings of these studies and proposes future avenues of research to investigate the effects of regular exercise on aspects of adaptive immunity in the elderly as they relate to the IRP. Copyright $\odot 2009$ S. Karger AG, Basel

\section{Introduction}

As a consequence of the exponential increase in life expectancy that has occurred in the developed world over the last century, the population is at an unprecedented risk of infectious diseases and malignancy due to apparently inevitable age-related declines in immunity. Immunosenescence, which contributes to poor vaccine efficacy and increased infection susceptibility in the elderly, is the term used to describe the biological aging of the immune

\section{KARGER \\ Fax +4161306 1234 \\ E-Mail karger@karger.ch}

www.karger.com
(C) 2009 S. Karger AG, Basel

$0304-324 X / 10 / 0565-0449 \$ 26.00 / 0$

Accessible online at:

www.karger.com/ger
Dr. Richard J. Simpson, BSc, PhD

Laboratory of Integrated Physiology, Department of Health and Human Performance University of Houston, Houston, TX 77204 (USA)

Tel. +1 713743 9270, Fax +1 7137439860

E-Mail rjsimpson@uh.edu 
Fig. 1. The frequency of individuals in the IRP category increases with advancing age, which is associated with an increased risk of infection due to changes in these categorized immune parameters. Whether or not habitual exercise and heightened physical fitness levels can influence the transition from non-IRP to IRP (and vice versa) remains to be established.

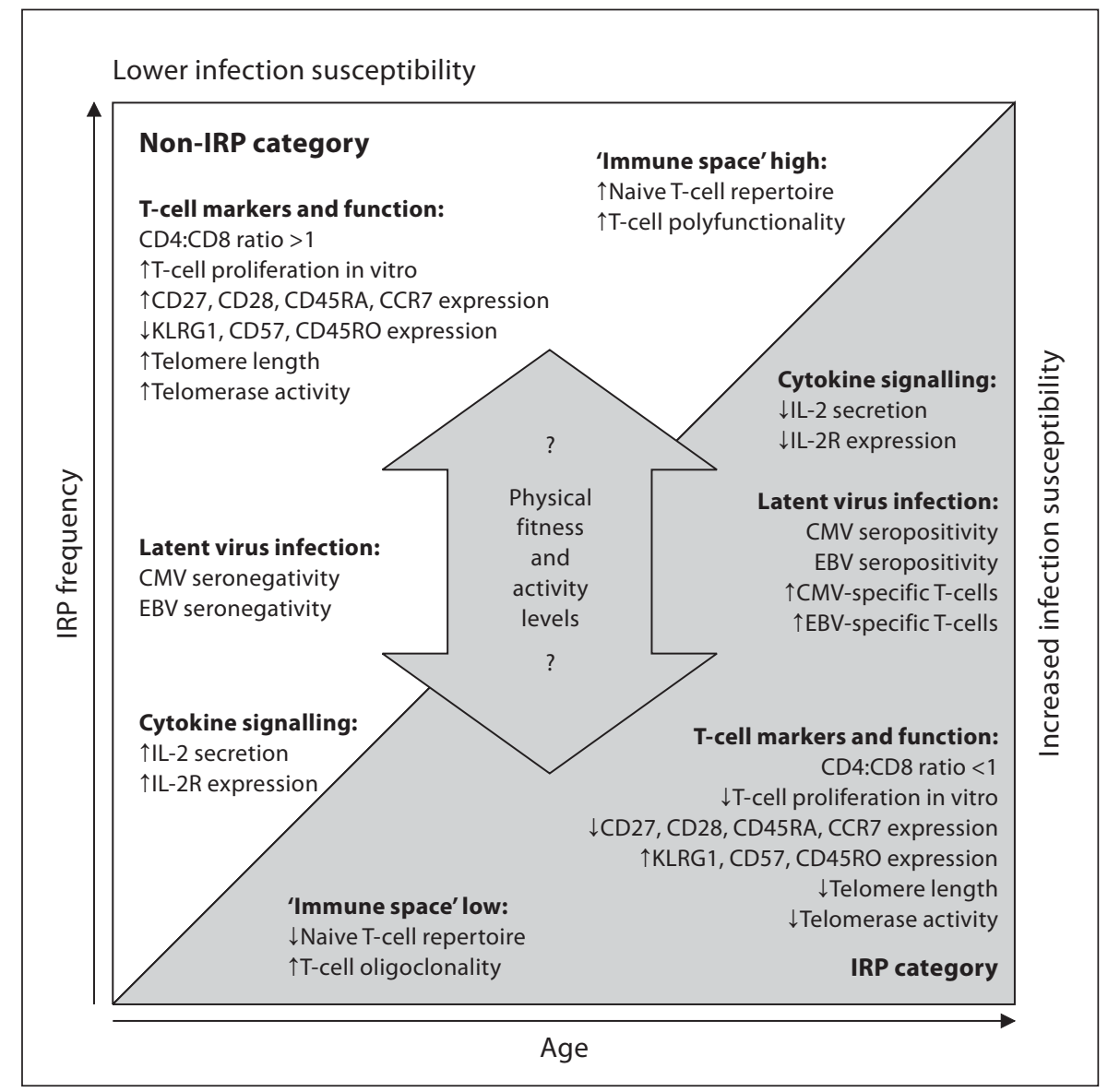

system. Although immunosenescence impacts on all components of the immune system, the deleterious effects of aging on innate immunity are not as clearly defined as the functional declines in adaptive immunity (i.e. T- and B-lymphocytes and their products) that are believed to make the greatest contribution to impaired immunity in older adults [1-3]. An array of immunological parameters has been identified which predict morbidity and mortality in the elderly; this has been coined the 'immune risk profile' (IRP) [1, 2]. Characteristics of the IRP include poor blood T-cell responses to mitogens and cognate antigens, a reduced naive $\mathrm{T}$-cell repertoire, an inverted $\mathrm{CD} 4+/ \mathrm{CD} 8+$ blood T-cell ratio, a greater frequency of T-cells expressing CD57 and the killer cell lectin-like receptor G1 (KLRG1), a lower frequency of T-cells expressing the co-stimulatory molecules CD27 and CD28, and the presence of antibodies against cytomegalovirus (CMV) in the plasma [1] (fig. 1).

Although the function of the immune system is known to decline with age in a manner that is perhaps akin to disease processes, some of the proposed interventions to combat immunosenescence (i.e. gene therapy, cytokine therapy) [4] in people who are not overtly ill raises a number of clinical and ethical difficulties. Other mechanisms have therefore been proposed to combat immunosenescence during aging, one of which is participation in a regular exercise regimen. It is generally accepted that middle-aged and elderly individuals who habitually participate in moderate-intensity exercise are less likely to incur an infection than their sedentary counterparts [5], the underpinning mechanisms perhaps being due to differences in some aspects of the IRP. It is not known, however, if exercise only acts in a preventative manner to delay immunosenescence or whether exercise has immune restorative properties in previously sedentary individuals. In this respect, two pertinent questions emerge. Firstly, can exercise delay, or even prevent an individual from making the transition from the non-IRP into the IRP category. And secondly, can regular exercise result in a previously sedentary individual 
reverting from the IRP to the non-IRP status (i.e. immune restoration). This review addresses the current knowledge on the effects of habitual moderate exercise on aspects of adaptive immunity as they relate to the IRP in older adults and discusses potential mechanisms and directions for future research.

\section{Exercise, Aging Immunity and the 'Immune Risk Profile'}

It is now generally accepted that habitual physical activity is associated with increased lifespan and lower disease risk in the elderly [5]. Despite many of these age-related conditions being linked to immunity, there is still a lack of understanding surrounding the potential mechanisms by which exercise might improve or maintain 'immune health' in older adults. Although the effects of acute exercise on immunity have been reasonably well studied in older adult populations, studies examining the impact of chronic exercise training are lacking. Although relatively few in number, these typically have been investigated using cross-sectional comparisons between active and inactive subjects or by use of randomized controlled trials incorporating an exercise intervention in previously sedentary older adults. Changes in T-cell number and phenotype, in vitro T-cell proliferation to mitogens, altered antibody titers in response to vaccination, changes in serum antibodies and NK-cell cytotoxicity are among the most popular dependent measures of immune function in response to habitual exercise [6]. Studies with a longitudinal experimental design tend to incorporate either an aerobic or resistance exercise intervention (or a mixture of both) ranging from 8 weeks to 12 months and have examined populations ranging from healthy middle-aged adults to frail elderly nursing home residents.

A problem with the existing literature is that the rationale for using a particular immune marker as an outcome measure to assess the impact of habitual exercise on aging immunity is not always clear. During the late 1990s, a longitudinal study in a very old Swedish population $(>85$ years) categorized an array of immunological parameters that could be used to predict mortality over 2, 4 and 6 years [7]. These measures were collectively coined the 'immune risk profile' (IRP), and have been adapted over the last decade to incorporate additional parameters from those initially identified. From the available literature to date, characteristics of the IRP in humans include: an inverted CD4: $\mathrm{CD} 8$ ratio $<1.0$; poor T-cell proliferation response to mitogens in vitro; increased numbers of CD8+
T-lymphocytes exhibiting an effector-memory T-cell phenotype; increased expression of the KLRG1 and/or CD57 on the surface of T-cells; lowered expression of the co-stimulatory molecules CD27 and/or CD28 on T-cells; latent CMV infection and, to a lesser extent, Epstein-Barr virus (EBV) infection; increased oligoclonality of virusspecific CD8+ T-cells that reduce the naive T-cell repertoire, and low IL-2 production and IL-2 receptor (IL-2R) expression [8] (fig. 1). The continued evolvement of the IRP serves to provide a useful criterion of immune parameters with which to assess the effects of regular exercise training in the elderly. We postulate that physical fitness and habitual exercise levels might influence the likelihood of an individual entering and reverting from the IRP category during late adulthood (fig. 1). Although no study to date has examined the impact of regular exercise on immunity in elderly individuals previously assigned to the IRP category, studies have been conducted to address some of the individual constructs that make up the IRP, albeit mostly involving otherwise healthy older adults. These individual constructs include changes in the CD4:CD8 T-cell ratio, changes in T-cell phenotype, in vitro T-cell responses to mitogens and alterations in IL-2 production and IL-2R expression.

\section{Exercise and the CD4:CD8 T-Cell Ratio}

An inverted CD4:CD8 ratio is associated with aging due to an accumulation of oligoclonal CD8+ T-cells, produced in response to persistent antigenic stimulation and/or age-associated increases in the resistance of CD8+ T-cells to apoptosis. Consequently, an inverted CD4:CD8 ratio of $\leq 1.0$ is usually indicative of a lowered naive T-cell repertoire and a diminished ability to respond to novel pathogens. Although an inverted CD4:CD8 ratio in the elderly is part of the IRP, it appears from the available literature that regular exercise has no effect on resting blood CD4:CD8 ratios in older adults. Yan et al. [9] found no effect of physical activity status on the CD4:CD8 ratio in men aged 20-73 years. Longitudinal exercise intervention studies have also found both aerobic and strength training exercise interventions to have no effect. Woods et al. [10] reported no change in the $\mathrm{CD} 4: \mathrm{CD} 8$ ratio in elderly subjects in response to 6 weeks of supervised aerobic exercise, although, interestingly, the mean resting CD4:CD8 ratio in the exercisers post-intervention was actually $<1.0$ [10]. Exercise interventions of 8 months in frail elderly have also failed to alter the CD4:CD8 T-cell ratio. Similarly, Fahlman et al. [11] and Flynn et al. [12] reported no change 
in response to 10 weeks of aerobic or strength exercise training respectively, while McFarlin et al. [13] also reported no change in resting $\mathrm{CD} 4+$ or $\mathrm{CD} 8+\mathrm{T}$-cell numbers in elderly women (aged 65-85 years) in response to 10 weeks' resistance training. Exercise intervention studies of longer duration have also reported no effect. Campbell et al. [14] examined the effects of a 12-month aerobic exercise intervention in older women (age 50-75 years) but found no effect on the CD4:CD8 T-cell ratio. Likewise, proportions of CD4+ and CD8+ T-cells have remained unchanged in response to 25 weeks [15] and 24 months of aerobic exercise training in healthy elderly [16]. Although exercise appears to have no effect on the CD4:CD8 ratio in apparently healthy older adults, it is important to note that none of these studies used subjects with CD4:CD 8 ratios $<1.0$ at baseline. Whether or not regular exercise can alter the CD4:CD8 T-cell ratio in people who are already in the IRP category remains to be determined. A recent study reported that elderly HIV-infected patients improved their CD4:CD8 ratios from 0.63 to 0.81 after 9 months' resistance exercise training [17]. A limitation of this work, however, was the lack of an appropriate control group, therefore it is difficult to determine whether the CD4:CD8 ratio increased due to the exercise intervention or some other factor such as an effective antiretroviral therapy [17].

\section{Changes in T-Cell Proliferation with Exercise}

Most cross-sectional studies report enhanced T-cell proliferation in physically active older adults compared to their sedentary counterparts. Conversely, longitudinal studies mostly report no change in T-cell proliferation in response to either an aerobic or resistance exercise training intervention. Nieman et al. [18] compared T-cell proliferative responses to PHA between aerobically conditioned and sedentary older women aged 65-85 years. Despite no differences in total T-cell number, T-cell proliferation was found to be greater in the aerobically trained women [18]. Similarly, Shinkai et al. [19] reported that T-cell proliferative responses to the mitogen PHA were $44 \%$ higher in a group of older (mean 63 years) recreational male runners in comparison to sedentary older men (mean 66 years) despite no differences in absolute T-cell numbers. It is important to note, however, that although T-cell numbers were similar between the active and sedentary groups in the studies by Nieman et al. [18] and Shinkai et al. [19], it remains possible that the proportions of naive, memory and terminally differentiated Tcells could have varied between these two subject groups altering the total $\mathrm{T}$-cell proliferative response to in vitro stimulation. The sedentary women in the study by Nieman et al. [18] were also subjected to 12 weeks of aerobic exercise training (30-40 min walking, 5 days/week) or flexibility exercise, but no intervention effect on T-cell proliferation was observed in either group despite a $13 \%$ increase in maximal oxygen uptake in the aerobic exercise group. Similarly, other longitudinal research studies have reported no effect of an exercise intervention on Tcell proliferative responses to mitogens $[12,14,20]$. Frail elderly nursing home residents had no improvements in T-cell proliferation after 32 weeks of an exercise intervention comprising both endurance and resistance type exercise [20] and, in response to resistance exercise alone, Flynn et al. [12] reported no changes in T-cell proliferation after 10 weeks' training in previously sedentary elderly women. It was proposed by Campbell et al. [14] that the reason most studies report no effect of an exercise intervention is due to small sample size (as a consequence of subject attrition) and relatively short interventions (10-32 weeks). To address this, they measured in vitro Tcell proliferation at baseline, after 3 months and on completion of a 12-month aerobic exercise intervention in 115 overweight/obese postmenopausal women aged 50-75 years [14]. Despite high retention rates (6\% dropout over 12 months) and increases in $\mathrm{VO}_{2 \max }$ of almost $14 \%$ in the exercise group (compared to no change in the stretching control group), exercise training was still found to have no effect on in vitro T-cell proliferation [14]. Only one longitudinal study has reported an effect of exercise on T-cell proliferation in the elderly. Woods et al. [10] reported that 6 months of supervised aerobic exercise (composed of brisk walking) conducted 3 times/week in elderly individuals aged 65 years enhanced lymphocyte proliferation when stimulated with multiple doses of the mitogens concanavalin A and PMA in vitro when compared to a control group who performed 6 months of stretching/toning exercise. A limitation of this work was the use of a whole blood assay and ${ }^{3} \mathrm{H}$-thymidine incorporation to assess T-cell proliferation, so it is not known which T-lymphocyte subset(s) responded to the exercise intervention.

\section{Effects of Exercise on T-Cell Phenotype}

The process of senescence causes many of the ageassociated changes in T-cell phenotype and function. Throughout life, naive antigen-virgin cells are gradually replaced with expanded clones of antigen-experienced ef- 
fector and memory T-cells that exhibit a late differentiation phenotype. The thymus gland atrophies rapidly up to the age of 35 years and only $10-15 \%$ of functional thymic tissue remains at age 50 [21]. This results in a reduced output of naive T-cells and a consequential lower ratio of naive to memory T-cells and, combined with the increased oligoclonality of the remaining T-cells (particularly for the CD8+ subset), reduces the repertoire of T-cells capable of responding to new and novel pathogens. An attractive potential mechanism by which exercise might exert its beneficial effects on aging immunity is to increase naive T-lymphocyte generation in the elderly, either by restoring thymic output and/or stimulating extrathymic T-cell maturation in other tissues (i.e. the liver or intestines) and consequently increasing the naive to memory T-cell ratio. Although no human studies to date have examined the effects of exercise on thymic or extrathymic function in the elderly directly, studies have attempted to assess the impact of regular exercise on naive T-lymphocyte development indirectly by examining the phenotype of T-cells in the periphery. Using CD45RA and CD45RO as naive and memory T-cell markers respectively, Woods et al. [10] reported that 6 months of supervised aerobic exercise (composed of brisk walking) conducted 3 times/week had no bearing on the numbers or proportions of CD4+ or CD8+ T-cells exhibiting naive and memory phenotypes in elderly individuals aged 65 years. Unfortunately, the use of CD45RA and CD45RO are considered very crude markers of naive and memory T-cells, due to the fact that CD45RA can be re-expressed by effector memory T-cells. Changes in more specific markers of T-cell senescence in response to exercise have only recently started to receive attention in the literature.

Recent cross-sectional work from our group showed that middle-aged men with a higher estimated maximal aerobic capacity $\left(\mathrm{VO}_{2 \max }\right)$ had a reduced frequency of KLRG1/CD57 double positive cells, KLRG1+/CD28 - cells and CD45RA-/CD45RO+ cells within both CD4+ and CD8+ T-cell populations than age-matched subjects who had a lower estimated $\mathrm{VO}_{2 \max }$ [unpubl. data]. Estimated physical fitness had no influence on the proportion of senescent T-lymphocytes in the younger group (age 20-30 years), which perhaps emphasizes the potential benefits of regular exercise on cellular immunity during the aging process. Only two longitudinal studies to date have concerned the effects of an exercise training intervention on T-cell surface expression of the co-stimulatory molecule CD28. Kapasi et al. [20] found no effect of 32 weeks' endurance and resistance exercise in frail elderly nursing home residents (age 87 years) on CD28, CD45RA,

Coupling Aging Immunity with a

Sedentary Lifestyle
CD45RO or HLA-DR (activated T-cell) expression within CD4+ or CD8+ T-cells. In contrast, Shimizu et al. [22] found increased numbers and percentages of CD4+ Tcells expressing CD28 after a 6-month supervised aerobic exercise program in elderly males and females aged 61-76 years. A limitation of this work, however, was the failure to document phenotype changes in CD8+ T-cells, which are more susceptible to oligoclonality and the acquisition of a senescent phenotype with age than CD4+ T-cells. Discrepancies between these two studies $[20,22]$ could be due to experimental differences in age and gender of the participants and exercise intensity. The participants in the study by Shimizu et al. [22] completed 30-min cycling exercise 5 times/week, whereas the frail nursing home residents examined in the study by Kapasi et al. [20] performed basic mobility exercises such as bathroom visits, walking, standing and wheelchair movements.

In order to gain a better understanding of the potential mechanisms by which exercise might improve immunity in the elderly, future exercise intervention studies should incorporate a more detailed immunophenotyping analysis of peripheral blood T-cells. For instance, it is possible to detect recent thymic emigrants in the periphery using T-cell receptor recombination excision circles (TRECs) and/or the surface marker CD103 [23]. Also, extrathymic T-cells believed to mature in the liver and intraepithelial layer of the intestines can be identified in peripheral blood based on their expression of a number of distinct cell-surface markers [24].

\section{Changes in IL-2 and IL-2-Receptor Expression with Exercise}

The activation and proliferation of T-lymphocytes during antigen recognition is dependent on the secretion of IL-2 and the expression of its receptors. The synthesis of IL-2 and expression of high affinity IL-2Rs is impaired with age and associated with the IRP in the elderly. Thymic involution and a reduction in the naive T-lymphocyte repertoire may account for the reduced frequency of Tcells that can produce and respond to IL-2 with advancing age. It appears from the available cross-sectional data that IL-2 production is greater in older habitual exercisers in comparison to their sedentary counterparts. Ogawa et al. [25] reported a greater number of CD8+ T-cells expressing IL-2 in elderly women who had been participating in an active walking program for 4 years in comparison to sedentary age-matched controls. Similar findings were made by Beshgetoor et al. [26], who found female masters ath- 
letes ( $>40$ years) to have more than double the numbers of CD8+ T-cells expressing intracellular IL-2 following mitogen stimulation in comparison to non-athletes of the same age. Interestingly, exercise had no effect on the numbers of CD4+ T-cells expressing intracellular IL-2 [25, 26]. Shinkai et al. [19] found greater concentrations of IL-2 in the supernatant of cultured PBMCs stimulated with LPS in elderly habitual runners compared to sedentary agematched controls. This increase in IL-2 secretion might have explained the greater T-cell proliferation that was observed in the older runners [19]. Another study reported that 24 months of aerobic exercise training in elderly women (age 62-86 years) resulted in an increased percentage of lymphocytes expressing intracellular IL-2, but not IL-4 or IFN- $\gamma$ in response to in vitro stimulation with $\mathrm{PMA} /$ calcium ionophore compared to a group of women who had not yet undertaken the exercise program [16]. Limitations of this study include the lack of pre-intervention measures between the test subjects and the failure to identify the lymphocyte subpopulations responsible for the increased IL-2 expression.

The ability of T-lymphocytes to become activated by an antigen is dependent on the interaction between IL-2 and its receptor, which triggers cellular growth, differentiation and formation of effector-memory T-lymphocytes. Surprisingly, chronic exercise-induced changes in T-cell surface expression of the IL-2R have received little research attention. One study reported that active elderly women (aged 60-98 years) had a greater surface expression of the IL- $2 \alpha$ chain receptor CD25 when stimulated with CD3 monoclonal antibodies in comparison to inactive control subjects [27]. In a longitudinal study however, Kapasi et al. [20] found that CD25 expression on CD4+ or CD8+ Tcells did not change with a 32-week exercise intervention in frail elderly, although it could be argued that the ability to upregulate IL-2-R expression following T-cell activation is more important to the immune response than basal expression levels of the receptor. Although the IL- $2 \beta$ chain receptor CD122 is believed to play a more important role in cell signaling than CD25 following the IL2/IL2-R interaction, no study to date has assessed T-lymphocyte expression of CD122 in response to chronic exercise in elderly humans. This might be useful in assessing whether or not regular exercise can stimulate extrathymic production of naive T-cells as these have been shown to express the $\beta$ chain but not the $\alpha$-chain of the IL-2R (CD25-/CD122+), in addition to having T-cell receptors predominantly composed of $\gamma \delta$ polypeptide chains [24].

\section{Immune Responses to Exercise in vivo}

Despite obvious cross-sectional differences between active and inactive elderly in a number of immune parameters that relate to the IRP, the evidence to suggest that exercise training in previously sedentary subjects can improve cellular adaptive immune responses in vitro is less compelling. Although it might appear from the in vitro work that exercise has limited immune restorative properties in previously sedentary elderly, longitudinal exercise intervention studies on markers of immunity in vivo are more encouraging, with the majority of these studies using vaccines or recall antigens to induce an immune response. Chin et al. [28] reported that a 17-week exercise intervention in frail elderly prevented the decline in delayed-type hypersensitivity skin test response against seven recall antigens that was observed in the non-exercising control subjects. Another study immunized older adults (aged $>64$ years) before and after a 10month aerobic exercise training intervention with a trivalent influenza vaccine [29]. It was found that those who completed the exercise, which consisted of three 25- to 30 -min exercise sessions per week at $65-75 \%$ heart rate reserve, had a greater mean fold increase in antibody titer to $\mathrm{H} 1 \mathrm{~N} 1$ and $\mathrm{H} 3 \mathrm{~N} 2$ strains of influenza A virus than the controls who did not change their physical activity patterns [29]. Furthermore, a 25 -week exercise training intervention was recently reported to enhance skin reaction to tuberculin-purified protein derivative and reduce serum IgG4 concentration in the elderly ( $>60$ years) without any changes in the non-exercising control subjects, indicating that moderate exercise facilitates T-helper 1 (Th1) immune responses in the elderly [15]. In response to a novel antigenic challenge, physical activity status also appears to improve immune responses in the elderly. Smith et al. [30] cross-sectionally assessed delayed type hypersensitivity skin response to the novel protein antigen keyhole-limpet hemocyanin $(\mathrm{KLH})$ in active and inactive elderly subjects (60-79 years) who were immunized against KLH via intramuscular inoculation 21 days earlier. The physically active elderly had higher anti-KLH IgM, IgG and IgG1 serum antibody concentrations and enhanced delayed-type hypersensitivity responses compared to the inactive elderly subjects.

Although randomized exercise controlled trials that use vaccine efficacy or recall antigens as outcome measures appear to suggest enhanced immunity in the elderly, it cannot be determined conclusively from these in vivo studies that chronic exercise in previously sed- 
entary elderly is having immune restorative effects. Indeed, whether or not a long-term exercise intervention is required to increase vaccine efficacy in the elderly has recently been challenged. Recent work by Edwards et al. $[31,32]$ has shown that an acute stressor, either through mental stimulation or physical exercise (aerobic and eccentrically biased muscle-damaging bouts of exercise), given immediately prior to influenza intramuscular vaccination increases serum antibody responses compared to control subjects. Results from these studies might indicate that certain in vivo (i.e. vaccine efficacy) immune responses to exercise in previously sedentary elderly occur due to transient immune perturbations as opposed to a permanent restoration of the immune system. It is possible, therefore, that the increased vaccine response in participants subjected to longitudinal exercise could be a short-term effect that is facilitated by a recent bout of exercise as opposed to the exercise intervention itself. Whereas a single bout of acute exercise might transiently enhance immunity in a way that increases vaccine efficacy in the elderly, the resulting effects from a single bout of exercise are unlikely to induce significant immune changes that could potentially move an individual from the IRP category to the non-IRP category. Future studies should attempt to investigate the time-course effects of regular exercise on vaccine efficacy in previously sedentary elderly as this will help determine whether these effects of exercise are due to permanent, substantive immune restoration or transient immune perturbations.

\section{Impact of Exercise on Leukocyte Telomere Length and Telomerase}

There is a large body of evidence to suggest chromosome telomere erosion during the aging process underpins cell cycle arrest in T-cells and associated immune dysfunction in the elderly. Leukocyte telomere length has been shown to predict morbidity and mortality in humans and, consequently, there has been recent interest on the effects of physical exercise on telomere length and telomerase activity [33-35]. Telomeres are DNA nucleoprotein complexes that form the physical ends of linear eukaryotic chromosomes. They erode progressively with each round of cell division during, for example, T-cell clonal expansion in response to persistent viral infections. Excessive telomere erosion triggers mechanistic pathways for senescence causing the cell to undergo proliferative arrest. Elderly individuals are known to have shorter leukocyte telomeres than their younger counterparts, presumably due to a greater exposure to pathogens over the course of a lifetime coupled with a reduction in naive T-lymphocytes as a consequence of thymic involution.

With regards to the effects of exercise, Cherkas et al. [33] found that blood leukocyte telomere length was positively correlated with self-reported scores of leisure time physical activity, even after correcting for age, gender, BMI and smoking history in a sample cohort of 2,401 twins. Differences in telomere length between active and inactive subjects were reported to be around $200 \mathrm{nt}$, which corresponds to 10 years of biological aging [33]. Similarly, Ludlow et al. [34] reported that 50- to 70-year-old subjects expending <990 kcal/week had shorter leukocyte telomeres than subjects expending between 991 and 2,340 $\mathrm{kcal} /$ week (determined by a self-reported physical activity survey), but no differences were found between the groups for telomerase activity, which is the reverse transcriptase that functions to synthesize telomeric DNA repeats. Only one longitudinal study has been conducted to date, but found no effect of exercise on leukocyte telomere length in middle-aged obese women who completed 6 months of supervised aerobic exercise, despite increases in maximal oxygen uptake, leukocyte antioxidant activity and a reduced BMI [35]. It is not known, however, if a reduction in mean telomere length would have occurred with exercise in a non-obese middle-aged and elderly group, as obese individuals are known to have shorter leukocyte telomeres than their lean counterparts [36] perhaps due to different mechanisms of telomere erosion than those occurring in normal aging.

It should be noted, however, that a fundamental flaw associated with all of these previous studies [33-35] is the use of mixed peripheral blood leukocytes to obtain an average telomere length measurement, which might lack specificity when it comes to determining the biological age of the immune system. Future research should consider the impact of regular exercise on telomere length in both CD4+ and CD8+ T-cells, as it appears to be the CD8+ T-cells that are predominantly affected by age and an acquisition of a senescent phenotype.

\section{Exercise and Cytomegalovirus Infection}

It has been argued that immunosenescence has an infectious component in the shape of persistent infection with the latent $\beta$-herpesvirus CMV $[1,2]$. CMV is an intermittently reactivating infection that persists predomi- 
nantly in cells of the myeloid lineage. Although the immune system is well equipped to control CMV reactivation within the immunocompetent host, complete eradication of the virus never occurs and the immune system has to constantly maintain effective CMV surveillance and containment throughout the lifespan. Ironically, CMV is assumed to be a 'harmless' infection as it is clinically asymptomatic in healthy hosts, although continued control of the virus requires strong T-cell responses that could exhaust their proliferative mechanisms during the aging process, thus contributing to immunosenescence and enhancing infection susceptibility in later life $[1,2]$. Surprisingly, no study to date has considered the effects of regular exercise on attenuating immunosenescence in individuals who are carrying a latent $\mathrm{CMV}$ infection. We hypothesize that exercise might exert beneficial effects on aging immunity in those infected with CMV via one or two mechanisms. Firstly, exercise might physically mobilize CMV-specific T-cells from the lymphoid compartment into the periphery leading to generation of naive T-cells. And secondly, habitual physical exercise might reduce the potential for CMV reactivation throughout the lifespan.

In relation to this first potential mechanism, we have shown that acute aerobic exercise mobilizes T-lymphocyte subsets that are KLRG1+, CD57+ and CD28-into the peripheral blood compartment in both young and older adults [37,38]. T-lymphocytes that are specific to a number of latent viruses including CMV and EBV have, in turn, been shown to exhibit this surface phenotype [39]. It has been postulated that excessive oligoclonal expansions of CMV-specific T-lymphocytes occupy the immunological space, which, through a looped feedback mechanism, limits maturation and production of naive T-lymphocytes and promotes thymic involution during the normal aging process [1]. These phenotypical distinct T-cell subsets that are mobilized into the blood by acute exercise might be specific for latent viruses such as CMV and EBV, suggesting that exercise might serve as a way to remove excess virus-specific T-cell clones from the peripheral lymphoid tissues (i.e. the immunological space). Although this mobilization of the T-cells by exercise is transient, persistent cell mobilization through regular exercise might 'make space' and stimulate thymic output or extrathymic maturation of naive T-cells through this previously proposed looped feedback mechanism (which is likely to involve IL-7 and/or growth hormone synthesis $[40,41])$, thus increasing the naive T-cell repertoire.

The second potential mechanism is that exercise might exert negating effects on CMV-induced immunosenes- cence due to its stress-reducing properties. A recent study showed that an inverted CD4:CD8 ratio and CD8+ Tcells lacking expression of CD27 and CD28 was more profound in adults working under stressful conditions [42]. It was also reported that the frequency of CD8+ Tcells lacking surface expression of CD27 and CD28 was associated with higher overnight urinary cortisol secretion [42]. While cortisol could be having a direct effect on the acquisition of a senescent phenotype in these workers, periods of stress are known to trigger a reactivation of latent CMV infection [43], which could lead to the premature acquisition of a senescent phenotype in blood Tcells.

It is not currently known, however, if virus-specific Tcells are mobilized into the blood by acute exercise or even if physical activity status and fitness levels have any bearing of the acquisition of a senescent phenotype during aging in individuals who have previously been infected with CMV. This is an important area to address because physical exercise could serve as an alternative and safer strategy to combat CMV-induced immunosenescence during aging than riskier techniques such as therapeutic vaccination, monoclonal antibody therapy and cytokine therapy, which have all been suggested as possible methods [4].

\section{Potential Mechanisms for Immune Enhancement due to Habitual Exercise}

Despite the well-known physiological changes and health benefits that are known to occur in response to habitual exercise in the elderly, there is a dearth of studies that have attempted to identify the underlying mechanisms of enhanced immunity by physical exercise. While we have suggested that a reduction in CMV reactivation and/or a physical mobilization and subsequent activation-induced cell death of oligoclonal T-cells by regular exercise might help increase the naive $\mathrm{T}$-cell repertoire and create 'immune space', there are many other mechanistic possibilities that could account for enhanced immunity by regular exercise. An obvious potential mechanism is for exercise to increase thymic mass in the elderly, possibly through increased IL-7 and/or growth hormone synthesis $[40,41]$. Surprisingly, no studies to date have examined the effects of exercise on IL-7 secretion or thymic function (i.e. frequency of recent thymic emigrant T-cells in the periphery) in older adults. Alternatively, we have postulated that exercise might stimulate extrathymic T-cell maturation in the elderly resulting in 
an increased frequency of $\gamma \delta$ T-cells but this, again, has yet to be investigated.

In addition to the potential changes within the immune compartment itself, immune enhancement might occur due to the modulatory effects that habitual exercise has on body fat distribution, lipid metabolism, plasma hormones, cholesterol and peripheral circulation that are known to change with age. Indeed, increasing body mass and plasma leptin concentrations are inversely correlated with leukocyte telomere length [36], while changes in the cholesterol content of plasma cell membranes can alter Tcell proliferative responses, MHC class I and II expression and subsequent antigen presentation to T-lymphocytes [44]. While the maintenance of healthy blood circulation with regular exercise might help preserve T-cell trafficking and increase immune surveillance in the elderly, the preservation of growth hormone synthesis with exercise might help prevent impaired immune responses that are associated with aging-induced growth hormone deficiency such as decreased thymocyte maturation and subsequent migration of naive T-cells into the periphery [41].

\section{Summary and Future Perspectives}

While it is clear from the available literature that habitual physical exercise is associated with improved immunity in the elderly, the evidence available to suggest that physical exercise can be used to facilitate immune restoration in previously sedentary elderly is lacking. It is often reported from cross-sectional data that markers of immunity such as T-cell responsiveness to mitogens, telomere length and the naive/memory T-cell ratio are better preserved in physically active individuals compared to their sedentary counterparts. In contrast, most longitudinal studies that have been conducted on previously sedentary elderly have failed to document any changes in similar immune parameters, indicating that exercise might have limited immune restoration properties in elderly individuals who have previously lived sedentary lifestyles. It is likely that the individuals used for these cross-sectional studies have adopted physical activity as an integral part of their lifestyles for many years and have been physically active for a significantly greater period of time than what is typically used for exercise training intervention studies (i.e. 8 weeks to 12 months), suggesting that habitual physical exercise during the normal aging process might help preserve immunity in a way that prevents an individual from falling into the IRP category in old age. Although exercise intervention studies mostly report no effect on a number of different immune parameters, it should be noted that the majority of these have used sedentary but otherwise healthy older adults, therefore, immune restoration with exercise might still be possible in individuals who are 'at risk'. Indeed, exercise might be more beneficial to diseased elderly and/or those already in the IRP category and an exercise intervention study in IRP elderly would be illuminating. Despite the inconspicuous evidence surrounding the use of an exercise regimen to restore immunity in previously sedentary but otherwise healthy elderly, there is more noticeable evidence to suggest that regular exercise is effective when immune responses in vivo are considered (i.e. vaccine response). However, whether this is a consequence of permanent immune restoration or a transient enhancement of immunity due to exercise remains to be determined. Indeed, rather than simply looking for an effect, future research should focus on elucidating some of the potential mechanisms that might underpin enhanced immunity with exercise in the elderly.

Although it might be argued from the available evidence that aging immunity coupled with a sedentary lifestyle leads to a state of immunological damage that is not reparable by a relatively short-term exercise intervention, the ability to make valid conclusions from these studies is confounded by the large inconsistencies among the experimental designs. These include variations in the age, gender, criterion outcome immune measures, initial health status of the participants (i.e. non-IRP elderly) and the intensity, frequency, modality and duration of the exercise intervention. Clearly, many of these experimental differences must be addressed before physical exercise can be used in earnest as an effective immune restorative strategy in the elderly.

References

1 Pawelec G, Koch S, Franceschi C, Wikby A: Human immunosenescence: does it have an infectious component? Ann NY Acad Sci 2006;1067:56-65.

-2 Koch S, Larbi A, Ozcelik D, Solana R, Gouttefangeas C, Attig S, Wikby A, Strindhall J, Franceschi C, Pawelec G: Cytomegalovirus infection: a driving force in human $\mathrm{T}$ cell immunosenescence. Ann NY Acad Sci 2007; 1114:23-35.

3 Derhovanessian E, Larbi A, Pawelec G: Biomarkers of human immunosenescence: impact of cytomegalovirus infection. Curr Opin Immunol 2009;21:440-445.

4 Koch S, Solana R, Dela Rosa O, Pawelec G: Human cytomegalovirus infection and $\mathrm{T}$ cell immunosenescence: a mini review. Mech Ageing Dev 2006;127:538-543. 
5 Leveille SG, Gray S, LaCroix AZ, Ferrucci L, Black DJ, Guralnik JM: Physical inactivity and smoking increase risk for serious infections in older women. J Am Geriatr Soc 2000; 48:1582-1588.

6 6 Haaland DA, Sabljic TF, Baribeau DA, Mukovozov IM, Hart LE: Is regular exercise a friend or foe of the aging immune system? A systematic review. Clin J Sport Med 2008; 18: 539-548.

7 Wikby A, Maxson P, Olsson J, Johansson B, Ferguson FG: Changes in CD8 and CD4 lymphocyte subsets, $\mathrm{T}$ cell proliferation responses and non-survival in the very old: the Swedish Longitudinal OCTO-Immune Study. Mech Ageing Dev 1998;102:187-198.

8 DelaRosa O, Pawelec G, Peralbo E, Wikby A, Mariani E, Mocchegiani E, Tarazona R, Solana R: Immunological biomarkers of ageing in man: changes in both innate and adaptive immunity are associated with health and longevity. Biogerontology 2006;7:471-481.

-9 Yan H, Kuroiwa A, Tanaka H, Shindo M, Kiyonaga A, Nagayama A: Effect of moderate exercise on immune senescence in men. Eur J Appl Physiol 2001;86:105-111.

10 Woods JA, Ceddia MA, Wolters BW, Evans JK, Lu Q, McAuley E: Effects of 6 months of moderate aerobic exercise training on immune function in the elderly. Mech Ageing Dev 1999;109:1-19.

- 11 Fahlman M, Boardley D, Flynn MG, Braun WA, Lambert CP, Bouillon LE: Effects of endurance training on selected parameters of immune function in elderly women. Gerontology 2000;46:97-104.

-12 Flynn MG, Fahlman M, Braun WA, Lambert CP, Bouillon LE, Brolinson PG, Armstrong CW: Effects of resistance training on selected indexes of immune function in elderly women. J Appl Physiol 1999;86:1905-1913.

13 McFarlin BK, Flynn MG, Phillips MD, Stewart LK, Timmerman KL: Chronic resistance exercise training improves natural killer cell activity in older women. J Gerontol A Biol Sci Med Sci 2005;60:1315-1318.

14 Campbell PT, Wener MH, Sorensen B, Wood B, Chen-Levy Z, Potter JD, McTiernan A, Ulrich CM: Effect of exercise on in vitro immune function: a 12-month randomized, controlled trial among postmenopausal women. J Appl Physiol 2008;104:1648-1655.

- 15 Okutsu M, Yoshida Y, Zhang X, Tamagawa A, Ohkubo T, Tsuji I, Nagatomi R: Exercise training enhances in vivo tuberculosis purified protein derivative response in the elderly. J Appl Physiol 2008;104:1690-1696.

-16 Drela N, Kozdron E, Szczypiorski P: Moderate exercise may attenuate some aspects of immunosenescence. BMC Geriatr 2004;4:8.

17 Souza PM, Jacob-Filho W, Santarem JM, Silva AR, Li HY, Burattini MN: Progressive resistance training in elderly HIV-positive patients: does it work? Clinics (São Paulo) 2008; 63:619-624.
8 Nieman DC, Henson DA, Gusewitch G, Warren BJ, Dotson RC, Butterworth DE, Nehlsen-Cannarella SL: Physical activity and immune function in elderly women. Med Sci Sports Exerc 1993;25:823-831.

19 Shinkai S, Kohno H, Kimura K, Komura T, Asai H, Inai R, Oka K, Kurokawa Y, Shephard R: Physical activity and immune senescence in men. Med Sci Sports Exerc 1995;27: 1516-1526.

20 Kapasi ZF, Ouslander JG, Schnelle JF, Kutner M, Fahey JL: Effects of an exercise intervention on immunologic parameters in frail elderly nursing home residents. J Gerontol A Biol Sci Med Sci 2003;58:636-643.

21 Steinmann GG, Klaus B, Muller-Hermelink HK: The involution of the ageing human thymic epithelium is independent of puberty. A morphometric study. Scand J Immunol 1985;22:563-575.

22 Shimizu K, Kimura F, Akimoto T, Akama T, Tanabe K, Nishijima T, Kuno S, Kono I: Effect of moderate exercise training on T-helper cell subpopulations in elderly people. Exerc Immunol Rev 2008;14:24-37.

23 McFarland RD, Douek DC, Koup RA, Picker LJ: Identification of a human recent thymic emigrant phenotype. Proc Natl Acad Sci USA 2000;97:4215-4220.

24 Torfadottir H, Freysdottir J, Skaftadottir I, Haraldsson A, Sigfusson G, Ogmundsdottir HM: Evidence for extrathymic T cell maturation after thymectomy in infancy. Clin Exp Immunol 2006;145:407-412.

25 Ogawa K, Oka J, Yamakawa J, Higuchi M: Habitual exercise did not affect the balance of type 1 and type 2 cytokines in elderly people. Mech Ageing Dev 2003;124:951-956.

$\longrightarrow 26$ Beshgetoor D, Arrues S, McGuire K: Effect of competitive training on $\mathrm{t}$-cell mediated immune function in Master's female athletes. Int J Sports Med 2004;25:553-558.

27 Gueldner SH, Poon LW, La Via M, Virella G, Michel Y, Bramlett MH, Noble CA, Paulling E: Long-term exercise patterns and immune function in healthy older women. A report of preliminary findings. Mech Ageing Dev 1997;93:215-222.

28 Chin APMJ, de Jong N, Pallast EG, Kloek GC, Schouten EG, Kok FJ: Immunity in frail elderly: a randomized controlled trial of exercise and enriched foods. Med Sci Sports Exerc 2000;32:2005-2011.

29 Kohut ML, Arntson BA, Lee W, Rozeboom K, Yoon KJ, Cunnick JE, McElhaney J: Moderate exercise improves antibody response to influenza immunization in older adults. Vaccine 2004;22:2298-2306.

30 Smith TP, Kennedy SL, Fleshner M: Influence of age and physical activity on the primary in vivo antibody and $\mathrm{T}$ cell-mediated responses in men. J Appl Physiol 2004;97:491-498.

31 Edwards KM, Burns VE, Allen LM, McPhee JS, Bosch JA, Carroll D, Drayson M, Ring C: Eccentric exercise as an adjuvant to influenza vaccination in humans. Brain Behav Immun 2007;21:209-217.
32 Edwards KM, Burns VE, Reynolds T, Carroll D, Drayson M, Ring C: Acute stress exposure prior to influenza vaccination enhances antibody response in women. Brain Behav Immun 2006;20:159-168.

33 Cherkas LF, Hunkin JL, Kato BS, Richards JB, Gardner JP, Surdulescu GL, Kimura M, Lu X, Spector TD, Aviv A: The association between physical activity in leisure time and leukocyte telomere length. Arch Intern Med 2008;168:154-158.

34 Ludlow AT, Zimmerman JB, Witkowski S, Hearn JW, Hatfield BD, Roth SM: Relationship between physical activity level, telomere length, and telomerase activity. Med Sci Sports Exerc 2008;40:1764-1771.

35 Shin YA, Lee JH, Song W, Jun TW: Exercise training improves the antioxidant enzyme activity with no changes of telomere length. Mech Ageing Dev 2008;129:254-260.

36 Valdes AM, Andrew T, Gardner JP, Kimura M, Oelsner E, Cherkas LF, Aviv A, Spector TD: Obesity, cigarette smoking, and telomere length in women. Lancet 2005;366: 662-664.

37 Simpson RJ, Cosgrove C, Ingram LA, Florida-James GD, Whyte GP, Pircher H, Guy K: Senescent T-lymphocytes are mobilised into the peripheral blood compartment in young and older humans after exhaustive exercise. Brain Behav Immun 2008;22:544-551.

38 Simpson RJ, Florida-James GD, Cosgrove C, Whyte GP, Macrae S, Pircher H, Guy K: High-intensity exercise elicits the mobilization of senescent $\mathrm{T}$ lymphocytes into the peripheral blood compartment in human subjects. J Appl Physiol 2007;103:396-401.

-39 Thimme R, Appay V, Koschella M, Panther E, Roth E, Hislop AD, Rickinson AB, Rowland-Jones SL, Blum HE, Pircher H: Increased expression of the NK cell receptor KLRG1 by virus-specific CD8 T cells during persistent antigen stimulation. J Virol 2005; 79:12112-12116.

40 Aspinall R, Mitchell W: Reversal of age-associated thymic atrophy: treatments, delivery, and side effects. Exp Gerontol 2008;43: 700-705.

41 Dardenne M, Smaniotto S, de Mello-Coelho V, Villa-Verde DM, Savino W: Growth hormone modulates migration of developing $\mathrm{T}$ cells. Ann NY Acad Sci 2009;1153:1-5.

-42 Bosch JA, Fischer JE, Fischer JC: Psychologically adverse work conditions are associated with CD8+ T cell differentiation indicative of immunosenescence. Brain Behav Immun 2009;23:527-534.

43 Sarid O, Anson O, Yaari A, Margalith M: Human cytomegalovirus salivary antibodies as related to stress. Clin Lab 2002;48:297-305.

44 Shaikh SR, Edidin M: Polyunsaturated fatty acids, membrane organization, $\mathrm{T}$ cells, and antigen presentation. Am J Clin Nutr 2006; 84:1277-1289. 